\title{
Cyclotron resonance studies of electron dynamics in ET charge transfer salts
}

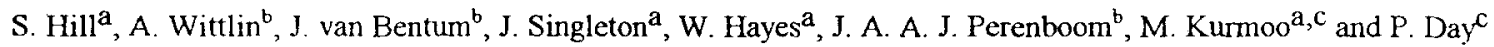

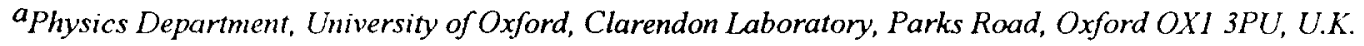 \\ ${ }^{b}$ High Field Magnet Laboratory, University of Nijmegen. Toemooiveld I, NL-6525-ED, Nijmegen, The Netherlands. \\ ${ }^{c}$ The Royal Institution, 21 Albemarle Street, London WIX ABS, U.K.
}

\section{$\underline{\text { Abstract }}$}

Microwave cyclotron resonance measurements on $(\mathrm{ET})_{2} \mathrm{NH}_{4} \mathrm{Hg}(\mathrm{NCS})_{4}$ have revealed a series of harmonics in addition to the fundamental absorption. An attempt is made to account for the associated cyclotron effective masses in terms of a renormalised band of quasiparticles.

\section{Introduction}

Organic charge transfer salts of the form $(\mathrm{ET})_{2}{ }^{+} \mathrm{X}^{\cdot}(\mathrm{ET}$ is short for BEDT-TTF or bis(ethylenedithio)tetrathiafulvalene) have attracted much interest recently due to the discovery of superconductivity in a number of what is now a large class of materials [1]. The mechanisms for superconductivity in these quasi-2D conductors is not well understood, although it has been suggested that an enhanced density of states (DOS) at the Fermi energy $\left(\varepsilon_{F}\right)$, brought about by electron-electron interactions (EEI), plays a key role [2]. A comprehensive study of the effective masses (a direct measure of the DOS) in these materials is therefore essential in order to test any potential theories. Cyclotron resonance (CR) is one technique which is used to measure effective mass and may also be used to detect any deviations from a simple parabolic band dispersion.

In a previous publication [3], we investigated $\mathrm{CR}$ in a series of ET salts $\left(\mathrm{X}=\mathrm{Cu}(\mathrm{NCS})_{2}, \mathrm{NH}_{4} \mathrm{Hg}(\mathrm{NCS})_{4}\right.$ and $\left.\mathrm{KHg}(\mathrm{NCS})_{4}\right)$. Cyclotron masses $\left(\mathrm{m}_{\mathrm{CR}}\right)$ were measured ranging from 0.4 to $1.2 \mathrm{~m}_{\mathrm{e}}$ in contrast to the effective masses obtained from the temperature dependence of the Shubnikov-de Haas (SdH) effect which were 2-5 times greater.

At this point, one needs to distinguish between different types of quasiparticle masses [4]. The effective mass $m^{*}$, which occurs in the thermodynamic density of states includes contributions arising from quasiparticles displacing each other as they move through a medium. The dynamical mass $m_{\lambda}$, is the mass which would be observed in the absence of quasiparticle interactions, and therefore represents the band mass renormalised only by electron-phonon interactions. The $\mathrm{SdH}$ effect examines oscillations in the electronic properties of a system as Landau levels cross the Fermi level. The effective mass measured in this way will therefore include effects due to EEIs, since it is at $\varepsilon_{\mathrm{F}}$ that these are strongest. In contrast, CR probes the energy separation between Landau levels at and above $\varepsilon_{\mathrm{F}}$. In the limit where the cyclotron energy $\left(\varepsilon_{\mathrm{CR}}\right)$ is much larger than the typical $\mathrm{EEI}$ bandwidth, $\mathrm{m}_{\mathrm{CR}}$ may be quite different from $\mathrm{m}^{*}$ (discussed below). Indeed, this is thought to be the reason for the difference in measured masses; $m_{C R}$ corresponds to $m_{\lambda}$, while the mass measured from the SdH effect is $m^{*}[4]$.
In the present work, we extend the previous study to lower energies in order to further investigate the relationship between the different quasiparticle masses. This paper will concentrate on the results for the (ET) $)_{2} \mathrm{NH}_{4} \mathrm{Hg}(\mathrm{NCS})_{4}$ salt which has the simplest Fermi surface (FS) of those studied, comprising a single closed 2D hole pocket as well as a pair of open $1 \mathrm{D}$ electron sections [5].

\section{Experimental details and results}

Two Klystrons (nominal frequencies 45 and $65 \mathrm{GHz}$ ), with approximately $5 \%$ frequency tuneability, were used as radiation sources. An array of ET crystals similar to those used in [3] were suspended within a cylindrical cavity; the Klystrons could then be tuned to a cavity resonance. Transmission through the cavity was observed by means of a carbon bolometer cooled together with the sample to $1.2 \mathrm{~K}$.

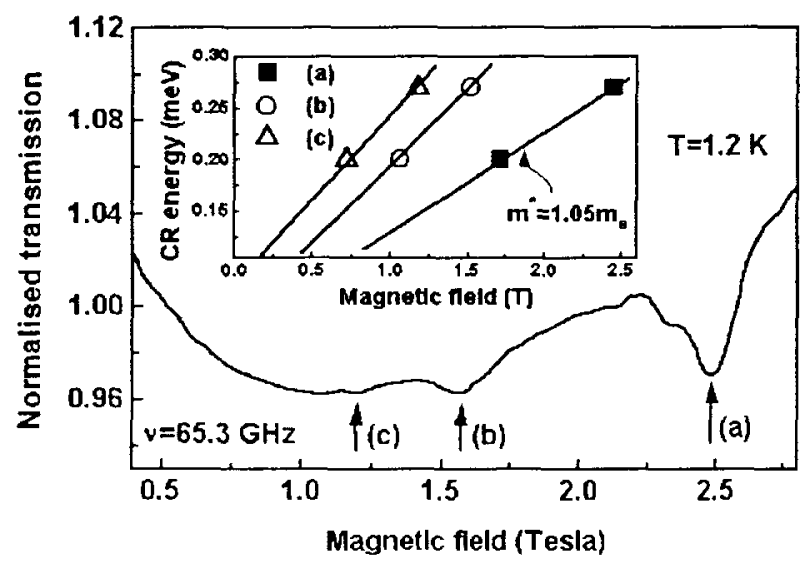

Figure 1. Transmission through $(\mathrm{ET})_{2} \mathrm{NH}_{4} \mathrm{Hg}(\mathrm{NCS})_{4}$. Inset shows the magnetic field position of features $a, b$, and $c$, for two cyclotron energies. 
Transmission through the cavity is plotted against magnetic field in Figure 1 for a frequency of $65.3 \mathrm{GHz}$. A series of features indicated by arrows is observed (labelled a to $c$ ). Similar results are obtained with the second Klystron. The frequencies at which the features (a) to (c) are observed are plotted against magnetic field position in the inset of Figure 1. Feature (a) is identified with the main CR which has previously been observed at magnetic fields $B>10 \mathrm{~T}$ [3]. However, the effective mass of $1.05 \mathrm{~m}_{e}$ associated with (a) is lower than the higher field value of $1.17 \mathrm{~m}_{e}$. Features (b) and (c) have not previously been observed and also behave in a way characteristic of CR.

The skin depth in metallic ET crystals is $\sim 1-10 \mu \mathrm{m}$ and so radiation is believed to couple to $C R$ via the sample edges on transmission through the array. This can be seen from the size of the resonances, which are 5-10 times larger than those previously observed at shorter wavelengths (see [3]). As the wavelength becomes smaller, a larger proportion of the incident radiation may pass through the array without interacting with a crystal. In the present case, the radiation is diffraction limited and so it may be assumed that the detected signal is well coupled to the sample via the cavity. Under these conditions, the exponential decay of the high frequency radiation field within the metal can lead to a relaxing of the conventional $C R$ selection rules if the skin depth is smaller than the characteristic cyclotron orbit [6]. It may then be possible to observe harmonics of the main CR transition, so called AzbelKaner resonances. This phenomenon is a likely candidate to explain the extra $\mathrm{CR}$ features at lower magnetic fields, since the single closed hole pocket in the FS of $(\mathrm{ET})_{2} \mathrm{NH}_{4} \mathrm{Hg}(\mathrm{NCS})_{4}$ is expected to yield only one CR. However, the cyclotron masses associated with (b) and (c) are $1.3 \mathrm{~m}_{e}$ and $1.4 \mathrm{~m}_{e}$ respectively, somewhat larger than the mass for the main transition (a).

\section{Discussion}

Any strong interaction between quasiparticles close to $\varepsilon_{F}$ will lead to an enhancement of the DOS and $\mathrm{m}^{*}$. The following discussion treats the case of a renormalised narrow band of quasiparticles about $\varepsilon_{F}$ and examines the consequences for Landau level quantisation and Fermiology.

A simple model can be used to re-calculate Landau levels based on what is known about quasiparticle bands close to $\varepsilon_{\mathrm{F}}$ in heavy Fermion compounds where EEIs are known to be particularly strong [7]. The model [8] assumes a quasiparticle mass $m_{\lambda}$ for all energies, except over a narrow energy band of width $\Delta_{l}$ about $\varepsilon_{F} ; \Delta_{l}$ characterises the bandwidth of the interaction. The model then assumes that the quasiparticle mass at $\varepsilon_{F}$ has a value which is consistent with $\mathrm{m}^{*}$ evaluated from the $\mathrm{SdH}$ effect $\left(\mathrm{m}^{*}=2.4 \mathrm{~m}_{e}\right.$ for $\left.(\mathrm{ET})_{2} \mathrm{NH}_{4} \mathrm{Hg}(\mathrm{NCS})_{4}\right)$. Provided that $\Delta_{\mathrm{l}}<<\varepsilon_{\mathrm{F}}$, the $\mathrm{SdH}$ oscillation frequency will remain unaffected, consistent with Luttinger's theorem [9]

Figure 2 shows a series of Landau levels renormalised in the manner described above; the straight lines represent the case of no renormalisation. The solid arrow indicates a typical $\mathrm{CR}$ transition for a quasiparticle at $\varepsilon_{\mathrm{F}}$, exciting it to the first unoccupied Landau level. This picture of CR is a little oversimplified; however, due to the enhanced DOS close to $\varepsilon_{\mathrm{F}}$, these transitions are likely to dominate the $\mathrm{CR}$ spectrum. In the limit $\varepsilon_{C R} \gg \Delta_{\mathrm{l}}$, the cyclotron mass will tend to $\mathrm{m}_{\lambda}\left(=1.17 \mathrm{~m}_{\mathrm{e}}\right.$ in $\left.(\mathrm{ET})_{2} \mathrm{NH}_{4} \mathrm{Hg}(\mathrm{NCS})_{4}\right)$. As $\varepsilon_{\mathrm{CR}} \rightarrow \Lambda_{\mathrm{I}}$ however, it is $\varepsilon_{\mathrm{CR}}$ which becomes enhanced by an amount $\delta$ (see figure 2), leading to a modified cyclotron mass:-

$$
\mathrm{m}^{\mathrm{n}} \mathrm{CR}_{\lambda}=\mathrm{m}_{\lambda}\left\{1-\delta / \mathrm{n} \times \varepsilon_{\mathrm{CR}}\right\},
$$

for the $n^{\text {th }}$ harmonic. This accounts for the lower fundamental $m_{C R}$ measured in the present study, since the mass is expected to decrease with decreasing energy. If we look at higher harmonic transitions, $m_{C R}$ is expected to become heavier, tending to $m_{\lambda}$ for successively higher harmonics. Although the data follows this trend, the CR masses are rather large compared with $1.17 \mathrm{~m}_{e}$ measured at higher magnetic fields. However, the above expression for $m_{C R}$ may be further offset when $\varepsilon_{C R}$ is comparable with or smaller than $\Delta_{\mathrm{l}}$, since $m_{C R}$ is expected to tend to the effective mass $\mathrm{m}^{*}$ in this limit.

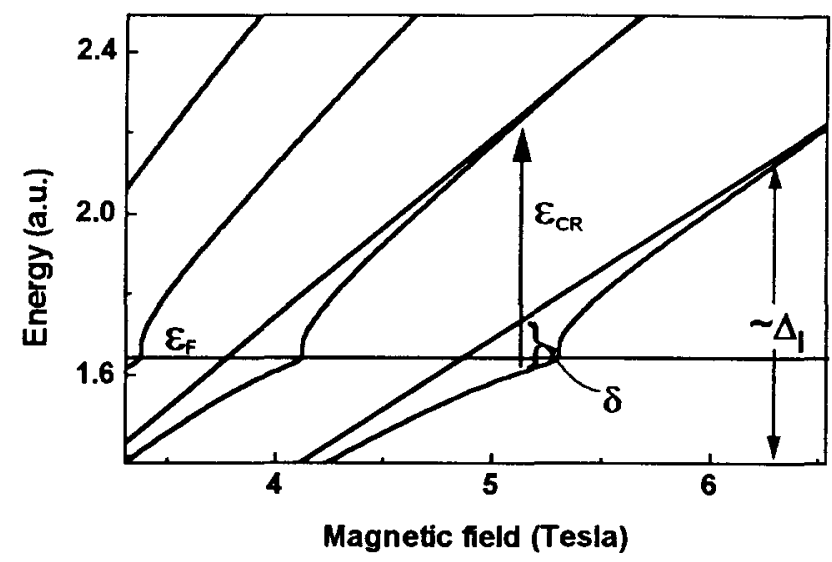

Figure 2. Renormalised Landau level scheme.

\section{Acknowledgements}

This work was supported by EPSRC and by the European Community. The Nijmegen Laboratory is supported by Stichting voor Fundamenteel Onderzoek der Materie (FOM) and by the European Community Large Installations Plan.

\section{References}

[1] T. Ishiguro and K. Yamaji (ed) 1990 Organic Superconductors (Berlin: Springer)

[2] J. Caulfield, W. Lubczynski, F. L. Pratt, J. Singleton, D. Y. K. Ko, W. Hayes, M. Kurmoo and P. Day, J. Phys.: Condensed Matter 6 (1994) 2911-2924

[3] S. Hill, J. Singleton, F. L. Pratt, M. Doporto, W. Hayes, T. J. B. M. Janssen, J. A. A. J. Perenboom, M. Kurmoo and P. Day, Synthetic Metals, 55-57 (1993) 2566-2571

[4] K. F. Quader, K. S. Bedell and G. E. Brown, Phys. Rev. B 36, 156 (1987)

[5] H. Mori, S. Tanaka, M. Oshima, G. Saito, T. Mori, Y. Murayama and H. Inokuchi, Bull. Chem. Soc. Jpn. 63, 2183 (1990)

[6] M. Ya. Azbel' and E. A. Kaner, Zh. Eksp. Teor. Fiz. 32. 896 (1956) [Sov. Phys. JETP 5, 730 (1957)]

[7] F. R. de Boer, J. J. Franse, P. H. Frings, W. G. M. Mattens, P. F. de Châtel, in High Field Magnetism edited by M. Date, North Holland, Amsterdam (1987) p 157

[8] S. O. Hill, PhD thesis, Clarendon Laboratory, Oxford (1994), Also to be published

[9] J. M. Luttinger, Phys. Rev. 1211251 (1961) 